\title{
KiloHertz Bandwidth, Dual-Stage Haptic Device Lets You Touch Brownian Motion
}

\author{
Tianming Lu, Cécile Pacoret, David Hériban, Abdenbi Mohand-Ousaid, \\ Stéphane Régnier, and Vincent Hayward, Fellow, IEEE
}

\begin{abstract}
This paper describes a haptic interface that has a uniform response over the entire human tactile frequency range. Structural mechanics makes it very difficult to implement articulated mechanical systems that can transmit high frequency signals. Here, we separated the frequency range into two frequency bands. The lower band is within the first structural mode of the corresponding haptic device while the higher one can be transmitted accurately by a fast actuator operating from conservation of momentum, that is, without reaction forces to the ground. To couple the two systems, we adopted a channel separation approach akin to that employed in the design of acoustic reproduction systems. The two channels are recombined at the tip of the device to give a uniform frequency response from $\mathrm{DC}$ to one $\mathrm{kHz}$. In terms of mechanical design, the high-frequency transducer was embedded inside the tip of the main stage so that during operation, the human operator has only to interact with a single finger interface. In order to exemplify the type of application that would benefit from this kind of interface, we applied it to the haptic exploration with microscopic scales objects which are known to behave with very fast dynamics. The novel haptic interface was bilaterally coupled with a micromanipulation platform to demonstrate its capabilities. Operators could feel interaction forces arising from contact as well as those resulting from Brownian motion and could manoeuvre a micro bead in the absence of vision.
\end{abstract}

Index Terms-Haptic interface design, dual-stage architecture, dynamic modeling, compensation.

\section{INTRODUCTION}

The human sense of touch is exquisitely sensitive to vibrations, and this over a significant band of frequencies reaching one $\mathrm{kHz}$ in healthy adult individuals [1]. Although the displacement detection threshold is function of numerous factors including finger temperature [2], contact condition [3], age [4], perceptual learning [5], hydration [6], health, for a review see [7], among numerous other factors, people remain through their lives extraordinarily sensitive to small oscillatory and transient mechanical perturbations, especially when applied to the hand. As a result, haptic interfaces ought to be able to transmit high frequency signals to the hand, lest the information that these signals contain be lost or tainted.

\subsection{Problem Addressed}

The transmission of high frequency movements is a longstanding problem in mechanical design. Owing to structural properties, any mechanical structure is subjected to an interplay between elastic properties and mass distribution.

- Tianming Lu, Stéphane Régnier, and Vincent Hayward are with the Sorbonne Universités, UPMC Univ Paris 06, UMR 7222, ISIR, F-75005 Paris, France

Email: $\{$ lu, regnier, hayward $\}$ @isir.upmc.fr.

- Cécile Pacoret is with the University of Geneva, department of Basic Neurosciences, Campus Biotech, 9 Chemin des Mines, 1205 Geneva, Switzerland

Email: cecile.pacoret@unige.ch

- Abdenbi Mohand-Ousaid is with FEMTO-ST Institute, AS2M department, Univ. Bourgogne Franche-Comté / UFC / CNRS UMR-6174 / ENSMM, 24, rue Alain Savary, 25000 Besanon, France

Email:abdenbi.mohand@femto-st.fr

- David Hériban is with Percipio Robotics, Maison des Microtechniques, 18, rue Alain Savary, 25000 Besançon, France

Email:david.heriban@percipio-robotics.com
Although it is an infinite dimensional system, in a frequency band a mechanical structure may be characterized by a finite number of modes that are reflected in its spectral response to excitation. The presence of mechanical modes corresponding to resonances and anti-resonances are generally undesirable for accurate motion transmission. Thus, structural engineers usually restrict the range of transmission of frequencies from static loading to an upper limit set well below the first resonant or anti-resonant mode.

A simple thought experiment makes the problem at hand quite evident. Take a $15 \mathrm{~cm}$-long, $5 \mathrm{~mm}$-diameter rod made of carbon fiber composite, the lightest and strongest material available today. The first mode of such a cantilevered beam is already at about $300 \mathrm{~Hz}$ and will get lower if anything is attached to the extremity.

A well accepted design wisdom aimed at increasing the mechanical bandwidth of an articulated structure calls for using parallel kinematic arrangements since the actuators can be grounded. These arrangements must nevertheless include cantilevered segments to achieve mobility. The result is that is hardly possible to design system with first modes at frequencies higher than one $\mathrm{kHz}$, unless these arrangements have very small workspaces. It is difficult to achieve a bandwidth greater than $300 \mathrm{~Hz}$ [8] and some highend devices exhibit modes as low as $30 \mathrm{~Hz}$ [9].

To overcome this problem we constructed a dual-stage haptic interface design that achieves a flat frequency response in the whole haptic range. Low frequency movements are transmitted by a conventional kinematic arrangement up to its first resonant mode. Above a few hundred Hertz vibratory amplitudes become small, falling below one millimeter. Thus, higher frequency oscillations can be reproduced by an inertia-based, ungrounded actuator at the tip 
operating on the basis of conservation of momentum. Using a crossover technique combined with dynamic modeling, identification, and frequency compensation, an overall flat response over the target range was achieved.

\subsection{Previous Works}

The literature on the design of haptic devices is abundant, so only some pertinent designs are mentioned here. The frequency response of a device, although it is a key factor of performance [14] [15], is rarely discussed, let alone measured, but a few numbers can be collected in the literature. These numbers are summarized in Table 1, as they are claimed by the corresponding references, although the method of measurement is rarely specified as recommended in [14] in order to ensure a level playing field.

Dual-stage robotic design can be traced a long way back. The research work [16] proposed a macro/micro manipulator architecture to enhance dynamic performance, but it was mainly focused on overall stability, accuracy improvement, and cycle-time reduction. The coarse-fine approach [17] proposed a novel parallel coarse platform combined with maglev device to obtain both large workspace and desirable force capability. However, the evaluation of overall haptic bandwidth was absent. The Distributed Macro-Mini ( $\left.\mathrm{DM}^{2}\right)$ method described in the research work [18] suggested a straight-forward parallel control structure to partition the input control torques into low and high frequency components based upon low frequency base actuator's low pass filter characteristics. However, this approach strongly depends on the dynamics of actuators for channel separation and further control scheme must be established in order to achieve uniform response over desired bandwidth. Moreover, this method was not validated experimentally. Before us some authors proposed to augment devices with tip-mounted vibrotactile actuators as in [19], but as far as we know, vibrotactile performance was the only factor which was tested. A single degree-of-freedom dual-stage device achieving a wide bandwidth was described in reference [20] [21] with a small motor coupled to a large one via a viscous transmission with the primary objective to reduce the overall apparent inertia. This approach, although effective to simultaneously improve several factors of performance, does not lend itself easily to be extended to multiple degrees of freedom. This is because achieving extra degrees of freedom implies adding additional structure, which will increase the overall apparent inertia and degrade the performance such as transparency and bandwidth. Other researchers developed high frequency devices taking advantage of feedback to extend the mechanical bandwidth as in [22]. However, new design trade-offs arose between performance and the number of degrees of freedoms since the integration of multiple degrees-of-freedom reintroduces the issues related to the transmission of high-frequency movements.

In this paper, a new technique is proposed that aims to extend the bandwidth of haptic devices with uniform magnitude by the addition of a vibrotactile crossover. Commercial devices as well as homemade devices keep their full DOF and workspace while their frequency is extended at few cost. This technique is demonstrated here with a Pantograph and a ready-to-use vibrotactile device.

\section{SYSTEM DESCRIPTION}

\subsection{Hardware}

The haptic interface design proposed here is based on a dual-stage architecture comprising a proximal and a distal stage.

The proximal stage is a Pantograph haptic interface [8], see Fig. 1a, which is based on a planar parallel mechanism with two actuated degrees-of-freedom. The closed chain structure with grounded motors, and a stiff, light-weight transmission design provide optimized dynamic performance. It was shown in [8] that the Pantograph is able to resolve 2D displacements of the order of $10 \mu \mathrm{m}$.

The distal stage is a vibration transducer, Fig. 1 (right), (Haptuator, Tactile Labs, Montréal, Canada) operating on the principle of conservation of momentum as described in [23]. This device comprises an internal moving magnet guided axially with respect to the case and actuated by a Laplace force acting between the magnet and the case. This actuator is efficient for high frequency and transient haptic feedback.

To couple these two stages to the load (user finger), the scheme given in Fig. $1 c$ is chosen among the several configurations described in [21]. Starting from the left, the Pantograph is grounded and coupled in series with the vibration transducer through an elastic element. In this part, the rectangular box and spring elements represent respectively the Pantograph's inertia and the elastic connection. Meanwhile, the vibration transducer, integrated inside the terminal assembly, is ungrounded and acts as an inertial actuator. Both stages are considered as a controllable source of force that acts on the last element of the scheme, the load. As a consequence of this arrangement, the Pantograph supplies the largest portion of rendered force covering low frequencies. While, the vibration transducer is responsible for transient effect covering high frequencies.
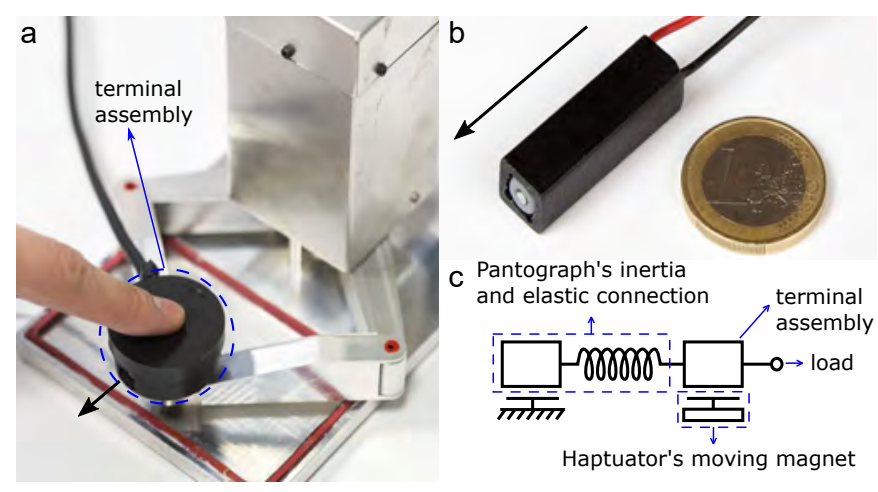

Fig. 1. System components and coupling scheme. The Pantograph (a) has a finger plate containing a vibrotactile actuator (b), oriented as indicated by the arrow. The finger plate also includes an accelerometer for monitoring. The coupling scheme is described by (c) [21].

The finger plate also included a three-axis MEMS accelerometer (MMA7361L, Freescale Semiconductors, Austin, TX, USA) to measure the signal transmitted to the finger on scale of $-6 \mathrm{~g}$ to $+6 \mathrm{~g}$. This sensor was used for system identification and was essential for evaluating the performance of the whole interface. The device was interfaced to a computer via a signal acquisition hardware (DAQ PCIe 6259, National 
TABLE 1

Frequency bandwidth of some haptic devices.

\begin{tabular}{lllllll} 
Model & Origin & DOFs & Workspace $[\mathrm{mm}]$ & Max. Force $[\mathrm{N}]$ & Bandw. $[\mathrm{Hz}]$ & Source \\
\hline Two finger grasp & Tech. Univ. Madrid & 6 & $100 \times 160$ & 3 & 8 & {$[10]$} \\
Freedom 7 & McGill University & 7 & $170 \times 220 \times 330$ & 2.5 & 30 & {$[11]$} \\
Phantom Desktop & Sensable & 3 & $160 \times 120 \times 120$ & 7.9 & 30 & {$[9]$} \\
Maglev & Butterfly Haptics, LLC & 6 & 24 & 40 & 75 & {$[12]$} \\
Pantograph & McGill University & 2 & $100 \times 160$ & 5 & 300 & {$[8]$} \\
ERGOS & Ergos Technologies & 1 & 50 & 60 & $20 \mathrm{k}$ & {$[13]$}
\end{tabular}

Instruments, Austin, TX, USA). The computer hosted a realtime Linux (RTAI) environment running signal processing algorithms at a $10 \mathrm{kHz}$ sampling rate.

\subsection{Dynamic Modeling}

For the system described above, a lumped parameter model of the system was developed, see Fig. 2. This model highlights the fact that the mechanical coupling of the two channels is more complicated than a mere acoustic summation, like in a network of loudspeakers. The system has three components, namely the vibration transducer-the Haptuator-a direct mechanical transmission - the Pantograph — and the finger of the user. Dynamic modeling was considered along one single axis, $x$, around a nominal position in the central region of the Pantograph's workspace, because the apparent masses depend on configuration. The parameters of the system are listed in Table 2.

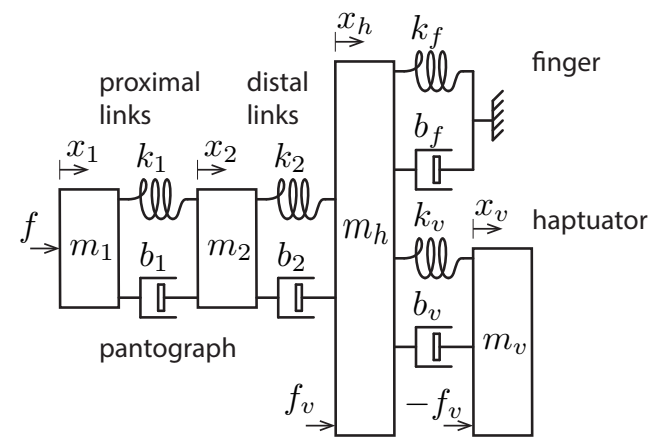

Fig. 2. System model. Lumped parameter model reflecting the main mass concentration, each corresponding to a mode.

The windings of the motors of the Pantograph apply a force, $f$, to the entire system via transmission shafts whose elasticity combines with those of the proximal links giving a combined elasticity parameter, $k_{1}$. The Haptuator produces a Laplace force, denoted by $f_{v}$ which is applied to the terminal assembly in contact with the finger. The force applied to the mass of the suspended magnet is thus $-f_{v}$. The magnet itself is guided by membranes modeled by elasticity and damping. The total force applied to the terminal assembly of mass, $m_{h}$, is thus the sum of the actions of the finger, the Haptuator's suspension, the Laplace force, and the distal links. All these effects can be collected in matrix form by,

$$
\boldsymbol{F}=\boldsymbol{M} \ddot{\boldsymbol{X}}+\boldsymbol{C} \dot{\boldsymbol{X}}+\boldsymbol{K} \boldsymbol{X}
$$

TABLE 2

Lumped mechanical parameters.

\begin{tabular}{ll} 
Parameters & Description \\
\hline$m_{h}$ & mass of terminal interface assembly \\
$m_{v}$ & mass of Haptuator's moving magnet \\
$m_{1}$ & combined mass of motor windings \\
$m_{2}$ & apparent mass of links \\
$k_{v}, b_{v}$ & stiffness and damping of Haptuator \\
$k_{1}, b_{1}$ & combined stiffness and damping of proximal links \\
$k_{2}, b_{2}$ & combined stiffness and damping of distal links \\
$k_{f}, b_{f}$ & stiffness and damping of finger [24] \\
$x_{h}$ & displacement of the terminal assembly \\
$x_{v}$ & displacement of moving magnet \\
$x_{1}$ & displacement of motors \\
$x_{2}$ & equivalent displacements of links
\end{tabular}

where

$$
\begin{aligned}
\boldsymbol{X} & =\left[\begin{array}{l}
x_{h} \\
x_{v} \\
x_{1} \\
x_{2}
\end{array}\right], \quad \boldsymbol{F}=\left[\begin{array}{c}
f_{v} \\
-f_{v} \\
f \\
0
\end{array}\right], \boldsymbol{M}=\left[\begin{array}{cccc}
m_{h} & 0 & 0 & 0 \\
0 & m_{v} & 0 & 0 \\
0 & 0 & m_{l} & 0 \\
0 & 0 & 0 & m_{2}
\end{array}\right], \\
\boldsymbol{C} & =\left[\begin{array}{cccc}
b_{2}+b_{v}+b_{f} & -b_{v} & 0 & -b_{2} \\
-b_{v} & b_{v} & 0 & 0 \\
0 & 0 & b_{1} & -b_{1} \\
-b_{2} & 0 & -b_{1} & b_{1}+b_{2}
\end{array}\right],
\end{aligned}
$$

and

$$
\boldsymbol{K}=\left[\begin{array}{cccc}
k_{2}+k_{v}+k_{f} & -k_{v} & 0 & -k_{2} \\
-k_{v} & k_{v} & 0 & 0 \\
0 & 0 & k_{1} & -k_{1} \\
-k_{2} & 0 & -k_{1} & k_{1}+k_{2}
\end{array}\right] .
$$

The system inputs are the torques applied to the motors lumped into a single signal, the desired haptic image expressed in workspace, and the output is the acceleration of the terminal assembly, $\ddot{x}_{h}$, or equivalently, the superficial skin layers of the finger in contact with the terminal assembly.

\section{Channel Separation, Identification and Compensation}

\subsection{Crossover Design}

The signal path begins with frequency separation by a filtering method borrowed from audio engineering known 
as a crossover. Crossovers can be optimally designed according to several criteria. A well-known crossover approach, widely applied to perform spectral partition, is the Linkwitz-Riley Filter. Such filters, constructed by cascading two Butterworth filters which have $-3 \mathrm{~dB}$ gain at cut-off frequency, are therefore characterized by $-6 \mathrm{~dB}$ gain at cutoff frequency. This means that compared to Butterworth crossover, it gives an overall amplitude response that is optimally flat and which is combined with good phase behavior [25]. Linkwitz-Riley filters are typically fourth order filters with slopes of $80 \mathrm{~dB} /$ decade. Figure 3 depicts the information flow in the system with the two-way crossover. The manner in which the signals recombine is not evident and thus merits further investigation.

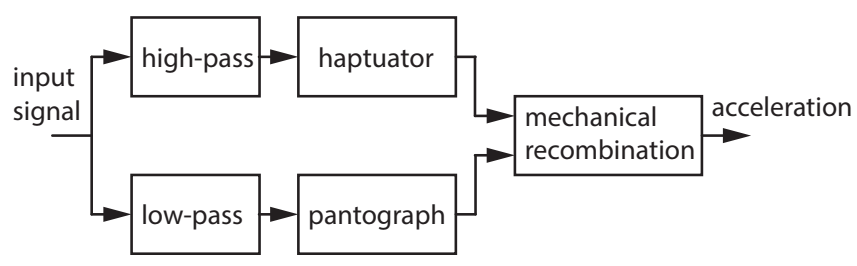

Fig. 3. System with crossover filters.

The crossover filters separate the signal into two paths with different frequency contents. Two different mechanical components receive the signals and recombine them to transmit movement to the finger interface. The whole system thus should behave like an all-pass, unity filter, i.e. the magnitude must be faithful to that of the original haptic signal [26]. Figure 4 shows the magnitude response of the Linkwitz-Riley fourth order crossover at pulsation, $\omega_{c}$, defined as follows,

$$
L(s)=\frac{\omega_{c}^{4}}{D(s)}, H(s)=\frac{s^{4}}{D(s)}, L(s)+H(s)=\frac{s^{4}+\omega_{c}^{4}}{D(s)},
$$

where

$$
D(s)=s^{4}+2 \sqrt{2} \omega_{c} s^{3}+4 \omega_{c}^{2} s^{2}+2 \sqrt{2} \omega_{c}^{3} s+\omega_{c}^{4} .
$$

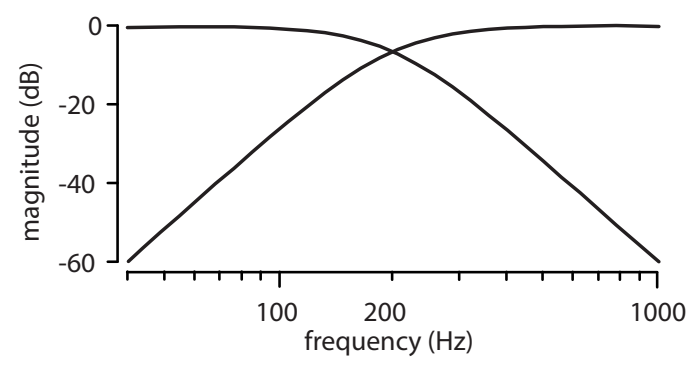

Fig. 4. Magnitude plot of the crossover's channels.

Because the Pantograph and the Haptuator share an overlapping frequency band, the cut-off frequency was selected to be the highest that the Pantograph could allow in order to minimize the delay introduced by the filters. The cut-off frequency must be chosen to satisfy all-pass filter requirement. This was achieved through identification as discussed next.

\subsection{Identification}

Our objective was to design a crossover method to separate the low and high frequency regions of haptic spectrum. The crossover method, Fig. 3, would be directly applicable insofar as the transducers could be assumed to have a flat response in their respective bands, which cannot be the case from the analysis of the previous section, see Fig. 2. It is therefore necessary to identify the responses of each transducer and cascade them with compensating filters.

The response of the low-frequency channel (activated Pantograph; inactivated Haptuator) was identified by measuring the Power Spectral Density (PSD) response to pseudo-random binary sequence input. Seen from Fig. 5, the original $300 \mathrm{~Hz}$ bandwidth [8] was reduced to $200 \mathrm{~Hz}$ in the present dual-stage arrangement, owing to the extra mass added at the tip.

The same method was applied to the high-frequency channel (inactivated Pantograph; activated Haptuator). This procedure was applicable as long as the system could be assumed to be linear and that the superposition principle applied. When coupled to the Pantograph, the Haptuator responds well to frequencies above $100 \mathrm{~Hz}$ and exhibits a smooth acceleration roll-off beyond $300 \mathrm{~Hz}$. At high frequencies, one of our ongoing study suggests that human perception does not discriminate between directions of movement, so only one distal actuator was needed.

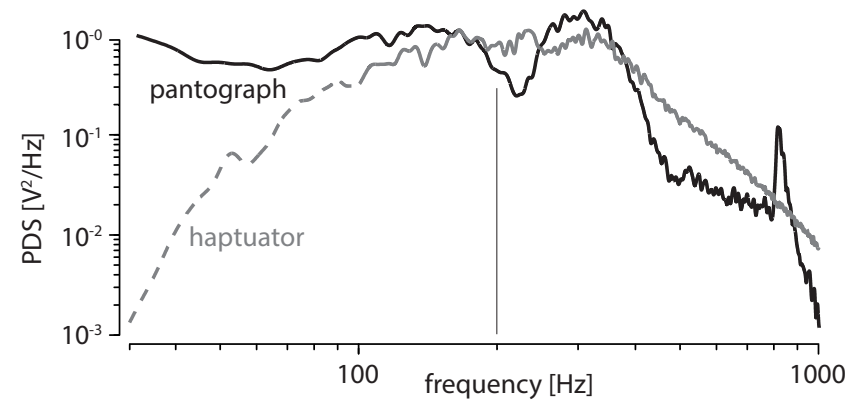

Fig. 5. Magnitude response from PSD analysis (Welch method).

In the frequency band from DC to $200 \mathrm{~Hz}$, the Pantograph had an acceptably uniform magnitude response. A first, nicely damped antiresonance is clearly visible after $200 \mathrm{~Hz}$. It is accompanied by a damped resonance at $300 \mathrm{~Hz}$. Another resonance-antiresonance pair is seem around $800 \mathrm{~Hz}$, but this time with a sharp peak. This behavior is explained by the model of Fig 2 with three masses connected by two damped springs [9]. Within the frequency band from DC to $200 \mathrm{~Hz}$, the Pantograph may be identified as a static gain. Its transfer function is then approximated by

$$
\widehat{G}^{\mathrm{L}}(s) \approx G_{0}^{\mathrm{L}}
$$

In the identification given with input signals only provided to the Haptuator, below $100 \mathrm{~Hz}$ the magnitude response (shown in dashed line) was not reliable since the device saturated with the motions of the internal moving mass exceeding its allowable range. For frequencies greater 
than $100 \mathrm{~Hz}$ the response was well approximated by the following model,

$$
\widehat{G}^{\mathrm{H}}(s) \approx \frac{250 s+6.4010^{5}}{s^{2}+2135 s+4.5610^{6}} .
$$

In the previous section, the lumped parameter model gave rise to an eight-order model but the consideration of a limited frequency band in the low frequency channel made it possible to ignore the high frequency modes, reducing the model complexity to a second order system. It can be observed from Fig. 5 that because of the interplay of masses and elasticities, the high frequency channel begins to roll-off at $300 \mathrm{~Hz}$. Thus, this channel must be compensated to achieve uniform magnitude. After analysis and identification, the scheme of Fig. 3 becomes that of Fig. 6 where, $L(s)$ and $H(s)$ are the Linkwitz-Riley filters, $C^{\mathrm{L}}$ and $C^{\mathrm{H}}$ the compensating filters, and $G^{\mathrm{L}}$ and $G^{\mathrm{H}}$ the high and low frequency transfer functions of the coupled system respectively.

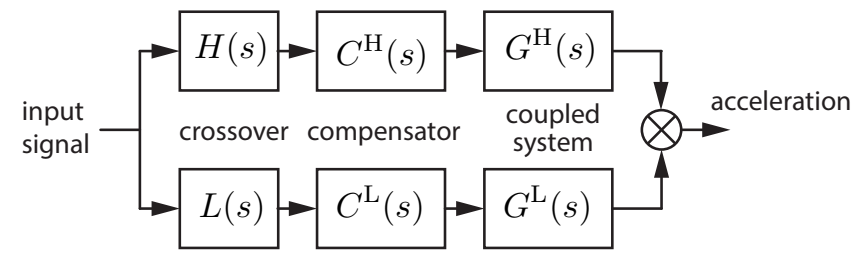

Fig. 6. System block diagram with compensation and crossover.

\subsection{Compensation}

The cross-over method assumes that the different channels have a uniform response in their respective bands, but that was not the case here. The channels had to be frequency and amplitude compensated in their respective bands for the haptic display fidelity that the magnitude of the transmission should not depend on frequency. From Fig. 5, it can be determined that the device had a sufficiently uniform magnitude response from DC to $200 \mathrm{~Hz}$. Hence, the only required compensation was to regulate the gain as follows,

$$
C^{\mathrm{L}}(s)=\frac{K_{\text {sys }}}{G_{0}^{\mathrm{L}}},
$$

where $K_{\text {sys }}$ stands for the desired overall gain and $G_{0}^{\mathrm{L}}$ is the estimated signal gain of the low frequency channel. The high frequency channel had a non-uniform magnitude response from $200 \mathrm{~Hz}$ to $1 \mathrm{kHz}$. Compensation for this channel could in principle be achieved by inversion of the identified model (2). Unfortunately, straightforward inversion would have resulted in a physically non-realisable filter with ever increasing magnitude in the high-frequencies. A simple solution was to perform a band-limited inversion according to

$$
C^{\mathrm{H}}(s)=K_{\mathrm{sys}} \frac{F(s)}{\widehat{G}^{\mathrm{H}}(s)},
$$

where $F(s)$ was a desired realizable response chosen here to be a second-order low-pass filter with a frequency roll-off starting at $1.0 \mathrm{kHz}$.

\subsection{Experimental Evaluation}

The real-time sampling rate was set to $10 \mathrm{kHz}$ and the acceleration magnitude response was measured for each third of octave in the frequency range of interest $(63,80$, $100, \cdots, 800,1000 \mathrm{~Hz})$. The results for the two individual channels and for the combined system are shown in Fig. 7. The system's response was indeed flat within a few $\mathrm{dB}$ from DC to $1000 \mathrm{~Hz}$.

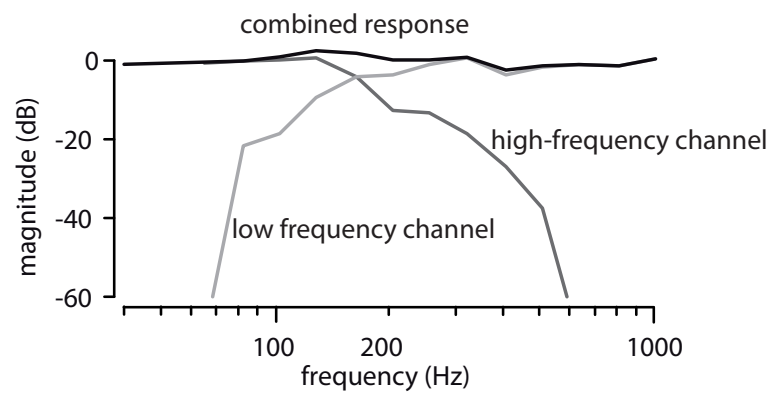

Fig. 7. The magnitude response of the combined interface.

\section{Interacting With The Micro-World}

To exemplify a new class of haptic interaction applications enabled by wide frequency haptic interfaces, we set up a micro-manipulation system where the manual interface was bilaterally coupled with an optical tweezer system [27].

Optical tweezers enable the manipulation of dielectric microscopic objects by steering them optically through a very steep axis-symmetric light intensity gradient produced by a highly focused laser. Bilateral tele-manipulation was enabled by simultaneously detecting the interaction force with the medium and surrounding objects through highspeed detection of the deflection of the microscopic objects away from their equilibrium position.

These instruments are very sensitive since the detected displacements can be as low as those induced by the ambient Brownian agitation and interactions forces can be detected in the picoNewton or nanoNewton range [28], [29], [30]. The user interface can be seen in Fig. 8. Here, we trapped a $3 \mu \mathrm{m}$ bead in water and displaced it to move into contact with a larger fixed object.

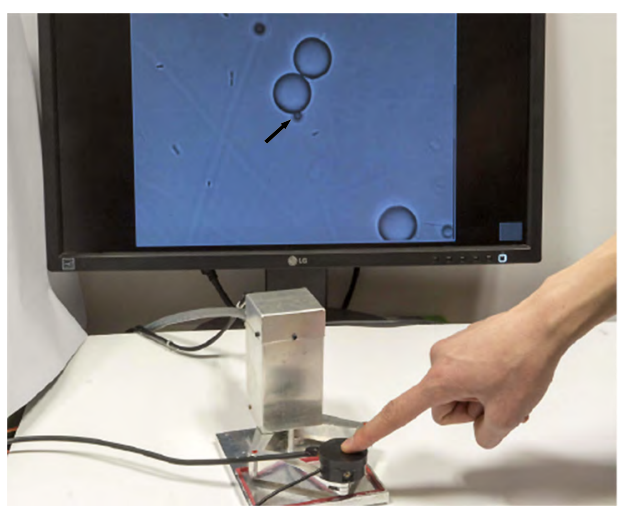

Fig. 8. Operator interacting with a $3 \mu \mathrm{m}$ glass bead (black arrow) via the dual-stage interface. Obstacles (bigger spheres) are $10 \mu \mathrm{m}$ glass bead attached to the coverslip. 


\subsection{Available Sensory Information}

In the entire haptic range from DC to one thousand Hertz, the various types of forces at play during the interaction with micro-beads could be experienced: thermal, viscous, and proximity Coulomb and Van der Vaals interaction forces. In particular, thermal and contact interactions have interesting high-frequency characteristics for bilateral haptic feedback.

When the trapped bead was in free space, as in Fig. 9a, thermal agitation was experienced. The thermal force is linked to the random collisions of the surrounding water molecules with the particle [27]. The following property is observed in accordance with the equipartition theorem at equilibrium:

$$
<\dot{x}(t)^{2}>=k_{B} T / m,
$$

Here $x(t)$ is the trajectory of the particle in one dimension relative to the equilibrium position define by the center of the optical trap. $m$ is its mass and $1 / 2 k_{B} T$ represent its thermal energy at an absolute temperature, $T$, and with $k_{B}$ the Boltzmann constant.

The theory of Brownian motion describes the instantaneous motion of the bead in a harmonic optical trap potential by the Langevin equation [31],

$$
m \ddot{x}=\left(2 k_{B} \gamma_{0} T\right)^{1 / 2} \eta(t)-\gamma_{0} \dot{x}(t)-\kappa x(t),
$$

given above in one dimension for simplicity. The Brownian interaction as a random force is necessary to obtain the equilibrium mention above. The stochastic process $\eta(t)$ is introduced to render the effect of the collisions with molecules of the surrounding medium. The effect of this fluctuating force can be summarized by this stochastic variable verifying a Gaussian distribution and :

$$
\langle\eta(t)\rangle=0 \text { and }\left\langle\eta\left(t_{1}\right) \eta\left(t_{2}\right\rangle=\delta\left(t_{1}-t_{2}\right), \forall t_{1}, t_{2} \in \mathbb{R} .\right.
$$

The other forces counterbalance the Brownian diffusion and maintain the particle in the laser trap: the fluid friction with $\gamma_{0}$ the fluid friction coefficient, and the harmonic force from the trap with $\kappa$ the trap stiffness.

Stokes's law for a spherical particle gives $\gamma_{0}=6 \pi \rho \nu R$, where $\rho, \nu$, and $R$ are the fluid density, fluid kinematic viscosity, and sphere radius, respectively. The characteristic time for the loss of kinetic energy through friction, $\approx 2.0 \times 10^{-6} \mathrm{~s}$, is three orders of magnitude shorter than our experimental time resolution so we can drop the inertial term and the governing equation becomes,

$$
\dot{x}(t)+2 \pi f_{\mathrm{c}} x(t)=(2 D)^{1 / 2} \eta(t),
$$

where the corner frequency of the damped optical trap (spring-damper system) is $f_{\mathrm{c}}=\kappa /\left(2 \pi \gamma_{0}\right)$ and $D=k_{B} T / \gamma_{0}$ is a diffusion constant.

Then, the particle displacement about the axis of the trap and its derivatives, see Fig. 9c, obey the form of a Gaussian distribution, with a mean value equal to zero and a variance that depends on the temperature, the trap stiffness, the fluid viscosity and other possible constraints.

Figure 9e shows the experimental histogram of the distribution of many samples read from the accelerometer which was mounted on the haptic handle, that is, the signal actually experienced by the user. This signal, indeed exhibiting
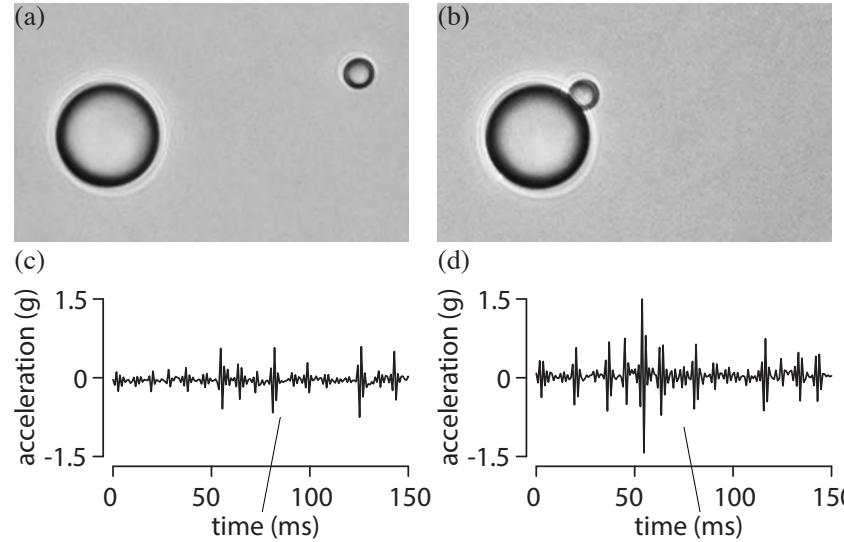

(d)

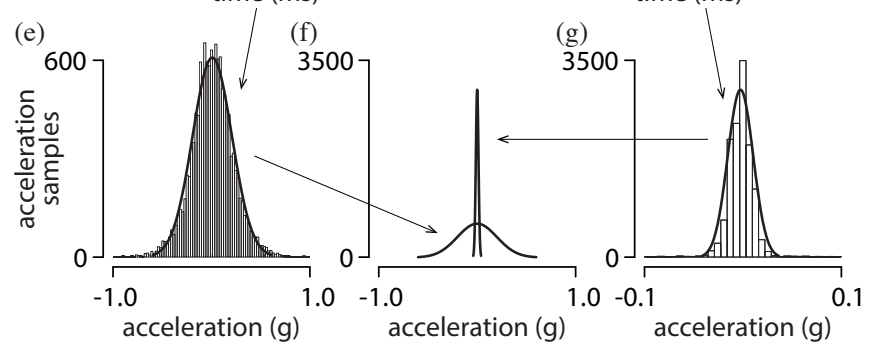

Fig. 9. Tangible differences between free space and contact in the micro world. (a) Manipulated bead in free space. (b) Bead brought into contact. (c) Molecular agitation felt through the interface. (d) Sharp transient at time $55 \mathrm{~ms}$ caused by a collision. (e) The distribution of acceleration magnitudes felt through the interface displays the hallmark of molecular interaction. (f) Dramatic reduction of agitation magnitude when the bead is pressed against an obstacle. (g) The distribution of acceleration magnitudes felt through the interface when the bead is pressed against an obstacle.

a characteristic Gaussian profile, is nothing but a magnified representation of the movements of the micro bead bombarded by thermally agitated water molecules. When the bead was moved around, a micro-collision occurred if an obstacle happened to be present in its path, Fig. $9 \mathrm{~b}$. This collision was felt as an acceleration spike that can be seen in Fig. 9 d.

At the vicinity of this fixed obstacle, the apparent fluid viscosity increased [27]. As a result, the variance of Brownian-induced agitation decreased and the distribution of acceleration due to molecular impacts became dramatically sharper as can be observed in the data collected in Fig. $9 f$.

These two high-frequency phenomena are experienced as tactile sensations by the operator helping her(him) to anticipate and adjust her(his) movements.

\subsection{Sensorimotor Manoeuvres in the Dark Micro World}

In order to assess the usability of the system, we asked five volunteers to manoeuvre micro-beads without the benefit of vision. Their task was to move a micro-bread about until they felt it to collide with an obstacle. At this point they had to move away from the obstacle in a direction normal to the surface on the basis of what they felt in the hand.

Inspection of Fig. 10 where five examples of trajectories are superposed onto a single image shows that the volunteers consistently succeeded at this task to a considerable degree. Trajectories that did not collide with any obstacle are not drawn in this figure. The ability to manipulate 
micro-objects when imaging is difficult shows considerable promise for many novel applications. Such as in [32] where objects are displayed under diffraction pattern or in [33] where target structures are too small to be resolved in the microscope image, effective haptics can be introduced to improve manipulation intuitiveness.

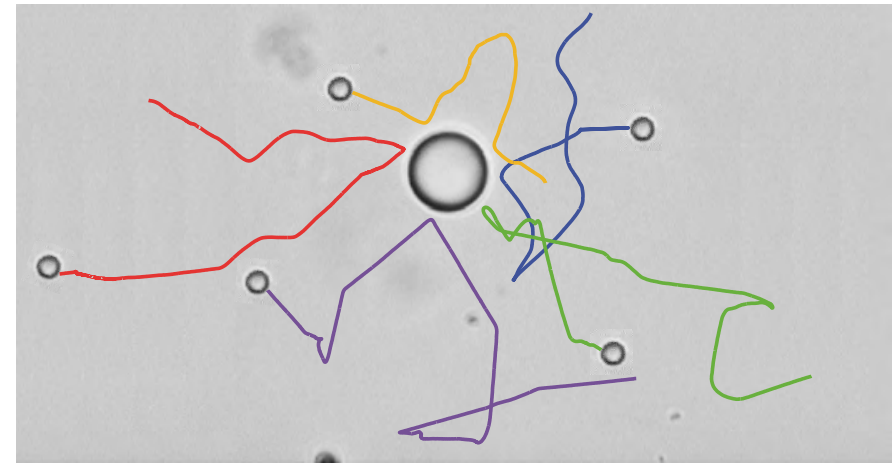

Fig. 10. Five examples of trajectories where volunteers blindly steered a micro-bead until it collided with an obstacle. The bead was then moved away from the obstacle.

\subsection{Preliminary Validation}

In order to validate the dual-stage approach experimentally, a set of experiments was designed as follows. Eight people, three females and five males, aged from twenty to thirty, volunteered to perform a test. They had limited knowledge and experience in haptics. They were asked to manipulate optical tweezers to circle around a spherical obstacle. The only instruction they were given was to maintain contact as long as possible between manipulated bead and obstacle. Each volunteer performed three sessions, each containing ten experimental trials. Each session had a different device configuration: in the first one, manipulation was performed under pure visual feedback (camera display of the scene) without haptics $(\mathrm{V})$; the second one with visual feedback and haptic feedback from the conventional Pantograph (P); and the third one with visual feedback and haptic feedback from the dual-stage interface (D). For simplicity, these three conditions will be abbreviated in the following text and figures as $\mathrm{V}, \mathrm{P}$, and $\mathrm{D}$ respectively. The session order was randomized, and the volunteers were not informed beforehand. Before the experiment, they were engaged in a training session of ten to fifteen minutes to get familiar with all three conditions. During each trial, if the manipulated bead escaped the laser trap due to volunteers' manipulation, e.g. too much force was exerted, or if the contact was not maintained for a significant portion of the trajectory (> 50\%), such manipulation would not be considered as successful and the related data were rejected.

Finally, the experiment was evaluated in terms of the number of successful trials, the average of the manipulation time per trial, the average force during trials, the average displacement error during trials. Results are plotted at the individual level in Fig. 11 and at the condition level in Fig. 12. It can be seen from Fig. 12 that the condition vision-only (V) has the lowest success rate, the longest manipulation time, the largest average exerted force and the highest displacement error. This means that in general the conditions with visual and haptic feedback ( $P$ and $D)$ have much better performance than the condition vision-only $(\mathrm{V})$. These results are highlighted in Fig. 12 on the number of successful trials and the displacement error which have significant difference according to a one-way ANOVA analysis on our small population (where assumptions of normality are not satisfied [34]). (a)
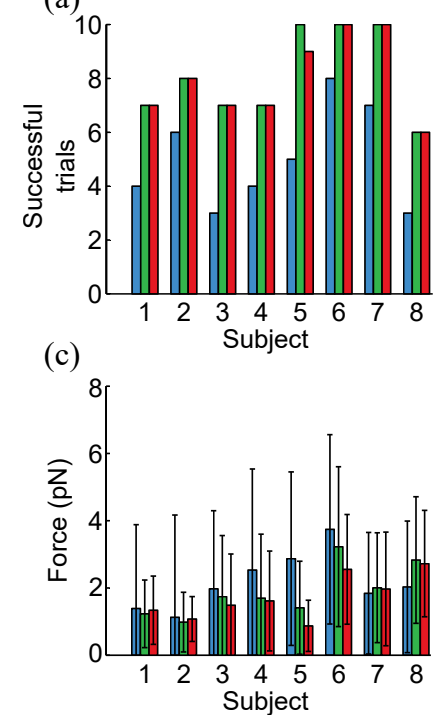

(b)

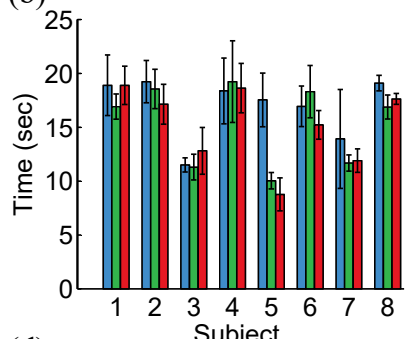

(d)

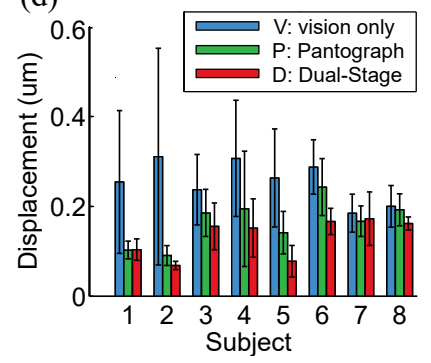

Fig. 11. Experiment results for each of the eight volunteers. The bars and whiskers represent the mean and standard deviation for each value respectively. (a) The number of successful trials. (b) The average manipulation time. (c) The average force. (d) The average displacement error. (V: visual feedback; P: visual feedback plus the Pantograph interface; D: visual feedback plus the dual-stage interface.)

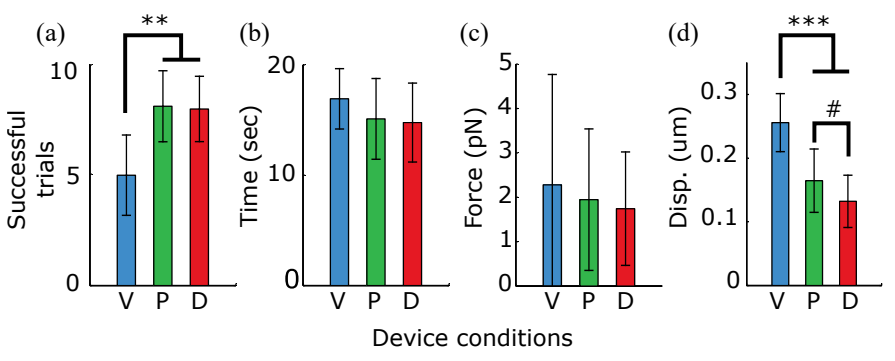

Fig. 12. Experiment results for each condition at the group level. The bars and whiskers represent the mean and standard deviation for each value respectively. The interesting statistical results from one-way ANOVA analysis are highlighted with: ${ }^{* * *} \mathrm{p}$-value $<0.005,{ }^{* *} \mathrm{p}$-value $<0.01$, and $\# \mathrm{p}$-value $=0.37$. (a) The number of successful trials. (b) The average manipulation time. (c) The average force. (d) The average displacement error. (V: visual feedback; P: visual feedback plus the Pantograph interface; D: visual feedback plus the dual-stage interface.)

The performance of the dual-stage device seems slightly better than the Pantograph in the tested configuration for maintaining an average force and minimizing the displacement error along the round trajectory. In Fig. 11d, it is interesting to note that the improvement due to the dualstage device is consistent across the subjects on the mean displacement error. The statistical tests do not reveal high significance, but show the potential for an improvement on the displacement error by the dual-stage device ( $p$-value $=0.37$, i.e. $63 \%$ probability of significant improvement) 
and motivate us for further investigation. This means that, when a task requires a low level of displacement error, the new device might be beneficial. A protocol that constrains user's strategy to minimize the displacement error could reveal this potential better than the present exploratory protocol. Further user studies should use this result in their experimental design and increase the tested population to study the gain on precision and accuracy due to the highbandwidth haptic devices.

\section{CONCLUSION AND FUTURE WORK}

This paper presented an approach to extend the frequency response of ordinary force-feedback haptic interfaces. The method consists of setting up a parallel structure with lowfrequency and high-frequency channels by signal crossover separation and using the intrinsic mechanics of a vibrotactile transducer to recombine the two signals paths. The combination of the low-frequency channel with the highfrequency channel realized by a recoil-type ungrounded high-frequency actuator results in a very simple design. Individual compensators could be implemented in order to equalize the overall magnitude response in frequency domain over the frequencies of interest to human perception. A key advantage of our approach is to enable the use of conventional force feedback devices for applications where high bandwidth response is desired. In our experiments, this large bandwidth haptic device has been tested in complex situations with several users. However, this first validation needs to be continued and further studies must investigate experimental designs with more specific instructions that constrain better user's exploration strategies. The impact on the displacement error promises interesting results when the trajectory accuracy and precision is important for the tasks, like avoiding delicate samples or contaminating agents. By rendering high dynamical interaction, better control is expected during a complex task such as an exploration at the dimension of live cells.

In future work, we plan to investigate the issues of closed loop stability of such systems which are immediately amenable to a channel separation approach [35]. We plan to extend this technique to several other force-feedback haptic devices.

\section{ACKNOWLEDGMENT}

The authors would like to thank Percipio Robotics for their contribution and support on this research project.

\section{REFERENCES}

[1] R. T. Verrillo, "Effect of contactor area on the vibrotactile threshold," The Journal of the Acoustical Society of America, vol. 35, no. 12, pp. 1962-1966, 1963.

[2] R. T. Verrillo and J. S. J. Bolanowski, "The effects of skin temperature on the psychophysical responses to vibration on glabrous and hairy skin," Journal of The Acoustical Society of America, vol. 80, no. 2, pp. 528-532, 1986.

[3] A. J. Brisben, S. S. Hsiao, and K. O. Johnson, "Detection of vibration transmitted through an object grasped in the hand," Journal of Neurophysiology, vol. 81, no. 4, pp. 1548-1558, 1999.

[4] R. T. Verrillo, "Age related changes in the sensitivity to vibration." Journal of Gerontology, vol. 35, no. 2, pp. 185-193, 1980.
[5] H. R. Dinse and M. M. Merzenich, "Adaptation of inputs in the somatosensory system," in Perceptual learning, M. Fahle and T. Poggio, Eds. MIT Press, 2002, pp. 19-42.

[6] R. T. Verrillo, S. J. Bolanowski, C. M. Checkosky, and F. P. McGlone, "Effects of hydratation on tactile sensation," Somatosensory E Motor Research, vol. 15, no. 2, pp. 93-108, 1998.

[7] T. Skov, K. Steenland, and J. Deddens, "Effect of age and height on vibrotactile threshold among 1,663 u.s. workers," American Journal Of Industrial Medicine, vol. 34, pp. 438-444, 1998.

[8] G. Campion, Q. Wang, and V. Hayward, "The Pantograph Mk-II: A haptic instrument," in Proceedings of the IEEE/RSJ International Conference on Intelligent Robots and Systems, IROS'05, 2005, pp. 723728.

[9] G. Campion and V. Hayward, "Fundamental limits in the rendering of virtual haptic textures," in Proceedings of the First Joint Eurohaptics Conference and Symposium on Haptic Interfaces for Virtual Environment and Teleoperator Systems, 2005, pp. 263-270.

[10] J. López, J. Breñosa, M. Galiana, I.and Ferre, A. Giménez, and J. Barrio, "Mechanical design optimization for multi-finger haptic devices applied to virtual grasping manipulation," Strojniski Vestnik/Journal of Mechanical Engineering, vol. 58, no. 7-8, pp. 431-443, 2012.

[11] V. Hayward, P. Gregorio, O. Astley, S. Greenish, M. Doyon, L. Lessard, J. McDougall, I. Sinclair, S. Boelen, X. Chen, J.-P. Demers, J. Poulin, I, Benguigui, N. Almey, B. Makuc, and X. Zhang, "Freedom-7: A high fidelity seven axis haptic device with application to surgical training," in Experimental Robotics $V$, ser. Lecture Notes in Control and Information Science, A. Casals and A. T. de Almeida, Eds., vol. 232. Springer Verlag, 1998, pp. 445-456.

[12] P. Berkelman and R. L. Hollis, "Dynamic performance of a magnetic levitation haptic device," in Intelligent Systems $\mathcal{E}$ Advanced Manufacturing, M. R. Stein, Ed. International Society for Optics and Photonics, 1997, pp. 140-149.

[13] J.-L. Florens, A. Luciani, C. Cadoz, and N. Castagné, "Ergos: A multi-degrees of freedom and versatile force-feedback panoply," in Proceedings of EuroHaptics, 2004, pp. 356-360.

[14] V. Hayward and O. R. Astley, "Performance measures for haptic interfaces," in Robotics Research: The 7th International Symposium, G. Giralt and G. Hirzinger, Eds. Heidelberg: Springer Verlag, 1996, pp. 195-207.

[15] S. D. Laycock and A. M. Day, "Recent developments and applications of haptic devices," Computer Graphics Forum, vol. 22, no. 2, pp. 117-132, 2003.

[16] A. Sharon, N. Hogan, and D. E. Hardt, "The macro/micro manipulator: An improved architecture for robot control," Robotics and computer-integrated manufacturing, vol. 10, no. 3, pp. 209-222, 1993.

[17] L. Stocco and S. E. Salcudean, "A coarse-fine approach to forcereflecting hand controller design," in Proceedings of the IEEE International Conference on Robotics and Automation, vol. 1, no. April. IEEE, 1996, pp. 404-410.

[18] M. Zinn, O. Khatib, B. Roth, and J. K. Salisbury, “Large workspace haptic devices-a new actuation approach," in 2008 Symposium on Haptic Interfaces for Virtual Environment and Teleoperator Systems. IEEE, 2008, pp. 185-192.

[19] S. A. Wall and W. Harwin, "A high bandwidth interface for haptic human computer interaction," Mechatronics, vol. 11, no. 4, pp. 371387, 2001.

[20] G. Millet, S. Haliyo, S. Regnier, and V. Hayward, "The ultimate haptic device: First step," in EuroHaptics conference, 2009 and Symposium on Haptic Interfaces for Virtual Environment and Teleoperator Systems. World Haptics 2009. Third Joint, March 2009, pp. 273-278.

[21] A. Mohand-Ousaid, G. Millet, S. Régnier, S. Haliyo, and V. Hayward, "Haptic interface transparency achieved through viscous coupling," International Journal of Robotics Research, vol. 31, no. 3, pp. 319-329, 2012.

[22] C. D. Lee, D. A. Lawrence, and L. Y. Pao, "A high-bandwidth force-controlled haptic interface," in Symposium on Haptic Interfaces for Teleoperation and Virtual Reality. ASME IMECE, 2000.

[23] H.-Y. Yao and V. Hayward, "Design and analysis of a recoiltype vibrotactile transducer," The Journal of the Acoustical Society of America, vol. 128, no. 2, pp. 619-627, 2010.

[24] M. Wiertlewski and V. Hayward, "Mechanical behavior of the fingertip in the range of frequencies and displacements relevant to touch," Journal of Biomechanics, vol. 45, no. 11, pp. 1869-1874, 2012.

[25] S. H. Linkwitz, "Passive crossover networks for noncoincident 
drivers," Journal of the Audio Engineering Society, vol. 26, no. 3, pp. 149-150, 1978.

[26] F. Harris, E. Venosa, P. Chen, Xi.and Muthyala, and C. Dick, "An extension of the linkwitz-riley crossover filters for audio systems and their sampled data implementation," in 20th International Conference on Systems, Signals and Image Processing (IWSSIP), 2013, pp. $175-178$.

[27] K. C. Neuman and S. M. Block, “Optical trapping," Review of Scientific Instruments, vol. 75, no. 9, pp. 2787-2809, 2004

[28] C. Pacoret, R. Bowman, G. Gibson, S. Haliyo, D. Carberry, A. Bergander, S. Régnier, and M. Padgett, "Touching the microworld with force-feedback optical tweezers," Optics Express, vol. 17, no. 12, pp. 10 259-10 264, 2009.

[29] Z. Ni, C. Pacoret, R. Benosman, and S. Régnier, "2d high speed force feedback teleoperation of optical tweezers," in Proceedings of the IEEE International Conference on Robotics and Automation, 2013, pp. 1700-1705.

[30] C. Pacoret and S. Regnier, "Invited article: A review of haptic optical tweezers for an interactive microworld exploration," Review of Scientific Instruments, vol. 84, no. 8, p. 081301, 2013.

[31] R. Kubo, M. Toda, and N. Hashitsume, Statistical Physics. Heidelberg: Springer Verlag, 1985, vol. 2.

[32] K. Kim, J. Yoon, and Y.-K. Park, "Simultaneous 3d visualization and position tracking of optically trapped particles using optical diffraction tomography," Optica, vol. 2, pp. 343-346, 2015.

[33] Y. Arai, R. Yasuda, K. Akashi, Y. Harada, H. Miyata, K. J. Kinosita, and H. Itoh, "Tying a molecular knot with optical tweezers," Nature, vol. 399, no. 6735, pp. 446-448, 1999.

[34] R. Hoekstra, H. Kiers, and A. Johnson, "Are assumptions of well-known statistical techniques checked, and why (not)?" Frontiers in Psychology, vol. 3, p. 137, 2012. [Online]. Available: http://journal.frontiersin.org/article/10.3389/fpsyg.2012.00137

[35] D. Prattichizzo, C. Pacchierotti, and G. Rosati, "Cutaneous force feedback as a sensory subtraction technique in haptics," IEEE Transactions on Haptics, vol. 5, no. 4, pp. 289-300, 2012.

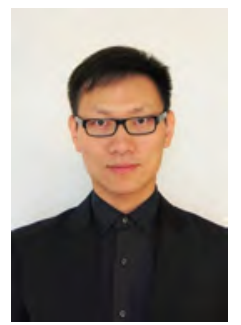

Tianming Lu received in 2007 the Bachelor's degree in Mechatronics from the Pilot Class (in collaboration with University of Michigan) at Shanghai Jiao Tong University, Shanghai, China. In 2010 he received the M.Sc. degree in Advanced and Robotic Systems from the University Pierre and Marie Curie, Paris, France, and the French Engineering degree (Diplôme d'Ingénieur) in Autonomous Robotics from the top-ranked French engineering school Ecole Nationale Supérieure de Techniques Avancées (ENSTA ParisTech), Paris, France. From 2010 to 2012, he worked as a robotics R\&D software engineer at Adept Technology France on algorithm development and real-time implementation for the new generation of the robotics controller Adept SmartController EX. He received in 2016 the Ph.D. degree in Robotics from the University Pierre and Marie Curie, Paris, France. His main areas of interest are robotics, planning algorithms, and haptics.

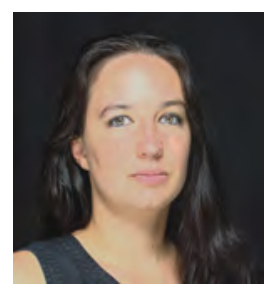

Cécile Pacoret received the M.Sc. in Robotics and Production Theory and the Mechanical Engineering degree from the top-ranked French Engineer School Art and Métier ParisTech in collaboration with University Pierre and Marie Curie, Sorbonne Universities, in Paris, France in 2007. She received Ph.D. degrees in Robotics from University Pierre and Marie Curie, Sorbonne Universities, in Paris, France in 2011. In 2012, she joined the Department of Mechanical Engineering, Carnegie Mellon University, as a Postdoctoral Fellow. She is currently since 2015 in the Department of Basic Neurosciences at Geneva University, as an associate researcher. Her main areas of research interest are human perception, neurosciences, robotics and biomimetic solutions.

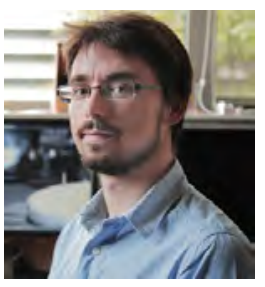

David Hériban is the founder and CEO of Percipio Robotics, a 5 years old French company specialized in micro robotic system design and production for industrial applications. He holds a Masters Degree in Mechanical Engineering from ENSMM (Micro Mechanical Engineering School) and a Masters Degree in Micromechatronics and Microrobotics from the University of Franche Comté. David worked for 4 years in FEMTOST Engineering Science Institute, to design and build a micromanipulation robotic system to handle 10 to $100 \mu \mathrm{m}$ sized objects. Then he has been trained in business development during 2 years to finally create the technological startup Percipio Robotics in 2011.

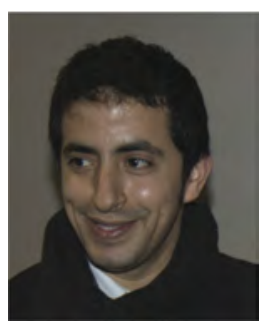

Abdenbi Mohand Ousaid received the Engineer degree in Automation from Ecole Nationale Polytechnique, Algiers, Algeria in 2008, an M.S. degree in Automation Signal and Images Processing from Paris 11 University, France in 2009 and a Ph.D. degree in Robotics in 2013 from the University Pierre and Marie Curie, Paris, France. From 2009 to 2014 he was a member of MICROB team of Institut des Systèmes Intelligents et de Robotique. In 2014/2015, he was a Temporary Assistant Professor at the University of Versailles, physics department, France $\mathrm{He}$ is currently an associate professor at the University of Franche-Comté/ department AS2M, FEMTO-ST institute. His researches interests are focused on the optimal and robust design of 3D micro mechatronics systems, micro actuation, micro-force sensing, additive manufacturing and 3D printing.

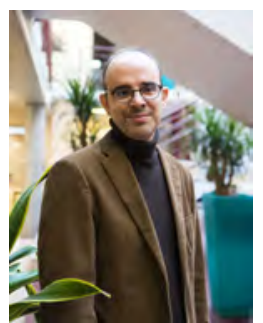

Stéphane Régnier received the Ph.D. degree in mechanical engineering and robotics from the University Pierre and Marie Curie, Paris, France, in 1996. He is full Professor at the Institute of Intelligent Systems and Robotics (ISIR), University Pierre and Marie Curie. Currently, he is Head of the ISIR micromanipulation team since 2001 and deputy vice president of research for the University Pierre and Marie Curie. His research interests include micro and nanomanipulation, teleoperation and haptic feedback at the nanoscale, micromechatronics, and biological cell characterization Stéphane Régnier has published more than 150 papers in journals and conferences. He is the associate editor for IEEE Transaction on Robotics and Journal of Micro-Bio Robotics.

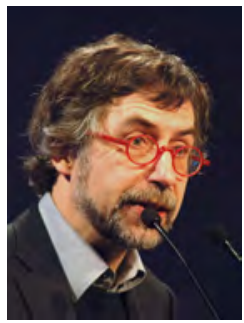

Vincent Hayward (M'84-SM'04-FIEEE'08), DrIng degree in 1981 from the University de Paris $\mathrm{XI}$. He was a postdoctoral fellow then a visiting assistant professor in 1982 at Purdue University, and joined CNRS, France, as Chargé de Recherches in 1983. In 1989, he joined the Department of Electrical and Computer Engineering at McGill University as an assistant, associate, and then a full professor in 2006. He is now professor at the Université Pierre et Marie Curie. He has published more than 250 articles in journals and conferences, co-founded spin-off companies and received best paper and research awards. He was on the editorial boards of the IEEE Transactions on Robotics, the ACM Transaction on Applied Perception, and the IEEE Transactions on Haptics. 